\title{
PII: SO038-1098(96)00453-X
}

\section{ELECTRONIC STRUCTURE OF RARE EARTH ARSENIDE/GALLIUM ARSENIDE SUPERLATTICES}

\author{
M. Said ${ }^{a, b}$, C.M. Bertoni ${ }^{a}$, A. Fasolino ${ }^{a, c}$ and Stefano Ossicini ${ }^{a}$ \\ ${ }^{a}$ Istituto Nazionale per la Fisica della Matcria (INFM) and Dipartimento di Fisica, Università di Modena, \\ 41100 Modena, Italy \\ ${ }^{b}$ Institut Preparatoire aux Etudes d'Ingenieur, 5019 Monastir, Tunisia \\ ${ }^{c}$ Dept. Theor. Physics I, University of Nijmegen, 6525 ED Nijmegen, The Netherlands
}

(Received 15 March 1996; in revised form 4 July 1996; accepted 15 July 1996 by E. Molinari)

\begin{abstract}
We present linear-muffin-tin-orbital calculations of the energy band structure and of the density of states of semimetal-conductor superlattices made of rare earth arsenide (ErAs and $\mathrm{YbAs}$ ) and $\mathrm{GaAs}$. The effect of size quantization and the possibility of a semimetal-semiconductor transition is studied by varying the number of rare earth arsenide monolayers buried in GaAs. We find that indeed a gap opens up in the band structure for the case of a single monolayer of $\mathrm{YbAs}$ embedded in GaAs, albeit well above the Fermi energy. The chances to induce a transition to a semiconductor are discussed. Copyright (C) 1996 Elsevier Science Ltd
\end{abstract}

Keywords: A. heterojunctions, A. nanostructures, D. electronic states.

There is a growing interest in the study of the electronic properties of rare earth arsenide (REAs) materials embedded in III-V compounds [1-6] due to several reasons: the possible development of metal-base transistors [2]: the relevant magnetic properties of REAs [3] (for example ErAs is an antiferromagnetic semimetal, with magnetism induced by the open $4 f$ shell); finally, it was argued that quantum confinement could led to a semimetal-semiconductor transition for very thin REAs layers [4]. A simple effective mass model predicts this transition with the opening of an energy gap of about $1 \mathrm{eV}$ for three monolayers of ErAs buried in GaAs [4]. However, the very first theoretical studies of these systems, based on tight binding calculations [5], ruled out this possibility for the case of $\mathrm{GdAs} / \mathrm{GaAs}$ superlattices (Gd has the same $6 s^{2} 5 d^{1}$ valence configuration as $\mathrm{Er}$ in ErAs) due to the crossing of conduction and valence bands of bulk GdAs at the $X$ point. A recent density functional calculation of the bulk band structure of ErAs and ErScAs [6] shows instead that the crossing is removed once quasiparticle energy corrections are included in a semiempirical way shifting upward the $d$ states of the RE atom. Therefore it is worth to reexamine the question of the electronic structure of REAs-GaAs superlattices by use of first principles approaches.

Here we report ab-initio calculations of the electronic properties of ErAs/GaAs and $\mathrm{YbAs} / \mathrm{GaAs}$ superlattices; in order to study quantum size effects we have considered different thicknesses of the REAs layer embedded in GaAs. Our main finding is that a gap opens up in the band structure, albeit above the Fermi energy. Thus the structure remains semimetallic; we will see that this is mainly due to the presence of interface states which may be sensitive to the structural interface arrangement. Therefore, our results are also relevant for the understanding of the growth mode of REAs deposited on GaAs.

In the calculations, we use the density functional theory in the local density approximation. We employ the Linear Muffin Tin Orbital (LMTO) method in the Atomic Sphere Approximation (ASA) including the combined correction term; empty spheres are included in the interstitial region and at the interface between REAs and GaAs. The details of the calculation and in particular the results for the whole series of REAs bulk compounds will be presented elsewhere [7]; here it is sufficient to note that our results for the density of states and band structures are in good agreement with those of [6] and [8] as far as the bulk properties of ErAs and YbAs are concerned.

The structures we considered for the superlattices are based on well matched lattices, grown along the $\left(\begin{array}{ll}0 & 0\end{array}\right)$ 
direction of the cubic structure. REAs preserves the sodium-chloride type structure with two type of atoms at each plane, whereas GaAs shows the zinc-blende stacking of 001 planes. We neglect the small lattice mismatch, which experimentally can be adjusted to some hundredth of a percent with adequate solid solutions [9-11], i.e. in the calculation all the atoms are located in their ideal positions. Concerning the configuration of the interface we adopt the geometry favoured by the experiments [11-13] with an As plane at each interface in the GaAs boundary; the As f.c.c. sublattice remains uninterrupted, with a stacking of 001 planes with $a / 2$ distance, whereas the $\mathrm{RE}$ and $\mathrm{Ga}$ sublattices are truncated at the interface. This choice, with As-reach interfaces, keeps the cell symmetrical with two identical interfaces and allows direct comparison with the previous calculations for GdAs/GaAs superlattices [14]. We have the following sequence of atomic planes: one plane of As atoms, two bilayers of GaAs, $N$ monolayers layers of REAs, two bilayers of GaAs. We refer to this structure as

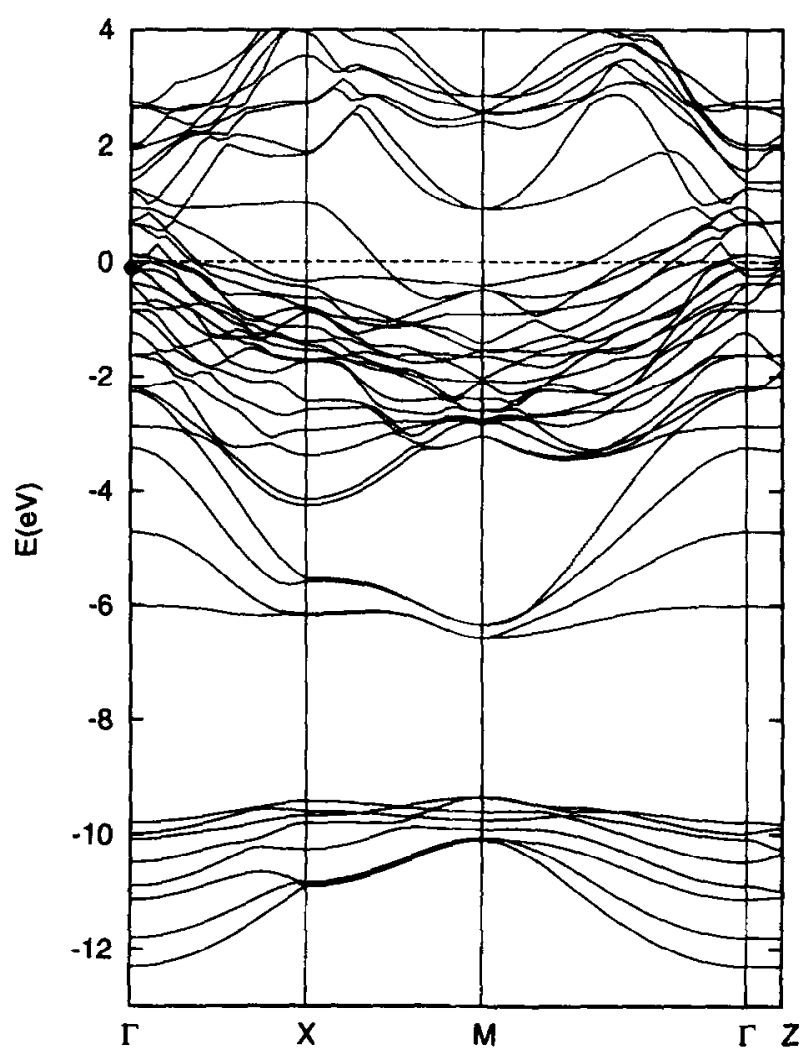

Fig. 1. Band structure of the $(\mathrm{As})_{1}(\mathrm{GaAs})_{2} /(\mathrm{ErAs})_{3} /$ $(\text { AsGa })_{2}$ superlattice projected along symmetry directions of the two-dimensional BZ of the $(100)$ surface and along the direction perpendicular to the surface $\mathrm{BZ}$, indicated as $\mathrm{Z}$. Energies (in $\mathrm{cV}$ ) are referred to the Fermi level of the system, showed by a dashed line. The dot shows the onset of the energy band discussed in the text.
$(\mathrm{As})_{1}(\mathrm{GaAs})_{2} /(\mathrm{REAs})_{N} /(\mathrm{AsGa})_{2}$. The height of the unit cell is $a[4+(N+1)] / 2$.

Figure 1 shows the energy band structures for the case ErAs/GaAs superlattice with $N=3$ along symmetry axes in the surface Brillouin zone (BZ) and along the direction $\Gamma-Z$ perpendicular to the surface $B Z$. All energies are given with respect to the Fermi level $\left(E_{F}\right)$ of the system. We see that the energy bands are not simply the superposition of the ErAs and GaAs bulk bands and that the superlattice is semimetallic; in particular we note an energy band with upward dispersion, starting near $\Gamma$ just below the Fermi energy (see the dot in Fig. 1), crossing the Fermi level in the $\Gamma-X$ direction, which originates mostly from the $d$ electrons of the $\mathrm{Er}$ atoms and two other bands located approximately $0.5-1 \mathrm{eV}$ above $E_{F}$ even in the $\Gamma-X$ direction, mostly of $p$ character and located on the As atoms at the interface. The band higher in energy, practically flat in this direction, bends downward in the $X-M$ direction and crosses $E_{F}$; the lower also crosses $E_{F}$ curving downward in the $\Gamma-X$ direction.

To understand better the nature and the location of these complicated and hybridized states we show in Fig. 2 the self-consistent projected density of states (PDOS) at the central ErAs monolayer, which is similar to the bulk one (dash-dotted line), at the ErAs layers at the interface (solid line), at the central (bulk) GaAs (dashed line) and at the GaAs layers at the interface (dotted line). In the low energy side, between $-12 \mathrm{eV}$ and $-10 \mathrm{eV}$, by comparing the As in REAs with respect to the As in $\mathrm{GaAs}$, we see that the peak, which is mostly related to the $s$-electron of As, is shifted up in energy by about $1 \mathrm{eV}$; the same shift is observed for the As $3 d$ core level spectrum from GaAs coated with 3.7 ML thick YbAs film [11], thus confirming the band alignment that comes

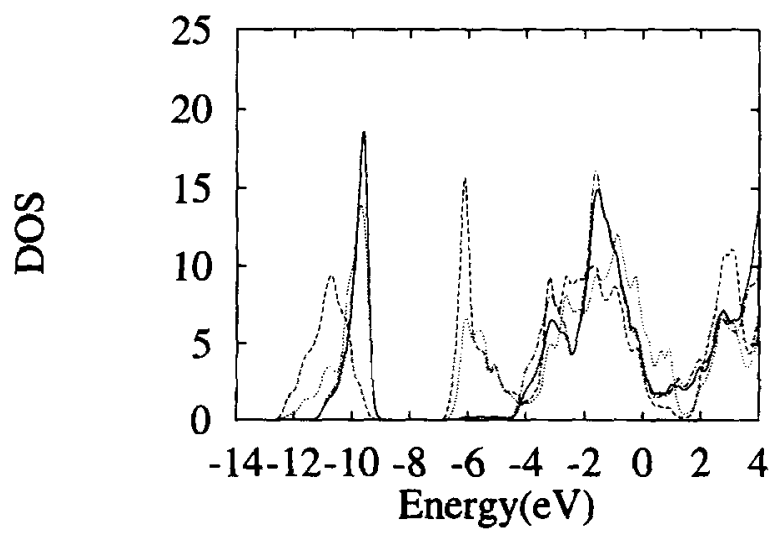

Fig. 2. Density of states of the (As) $)_{1}(\mathrm{GaAs})_{2} /(\mathrm{ErAs})_{3} /$ (AsGa) $)_{2}$ superlattice projected on the different sublattices: the central ErAs layer (dash-dotted line), the interface ErAs layer (solid line), the central GaAs layer (dashed line), the interface GaAs layer (dotted line). Energies (in $\mathrm{eV}$ ) are referred to the Fermi level of the system. 
out from our calculation. Near and above the Fermi level we see that the most important contributions to the DOS are two small peaks due to GaAs at the interface. By looking at the character of these states, we can identify them as due to interface states of $p$ character located on the As atoms at the interface. In fact, with the chosen structural interface arrangement, the interface As atoms have dangling bonds which give rise to interface states which pin Fermi level. Their nature of interface states is also witnessed by the finite dispersion along the $\Gamma-Z$ direction (see Fig. 1), the growth direction of the superlattices; these states are not due to the confinement of the REAs in GaAs. Similar interface states have been derived for GdAs/GaAs superlattices [5].

To examine the role of confinement we have performed calculation for the case of ErAs passing from $N=3$ to $N=1$. Figure 3 shows the energy band structure for $(\mathrm{As})_{1}(\mathrm{GaAs})_{2} /(\mathrm{ErAs})_{1} /(\mathrm{AsGa})_{2}$. We see that now the energy band with upward dispersion does not cross the Fermi level but starts $\sim 1 \mathrm{eV}$ above $E_{F}$ at the $\Gamma$ point. The relative density of states displays no gap but has a pronounced minimum at this value [7]. This is an

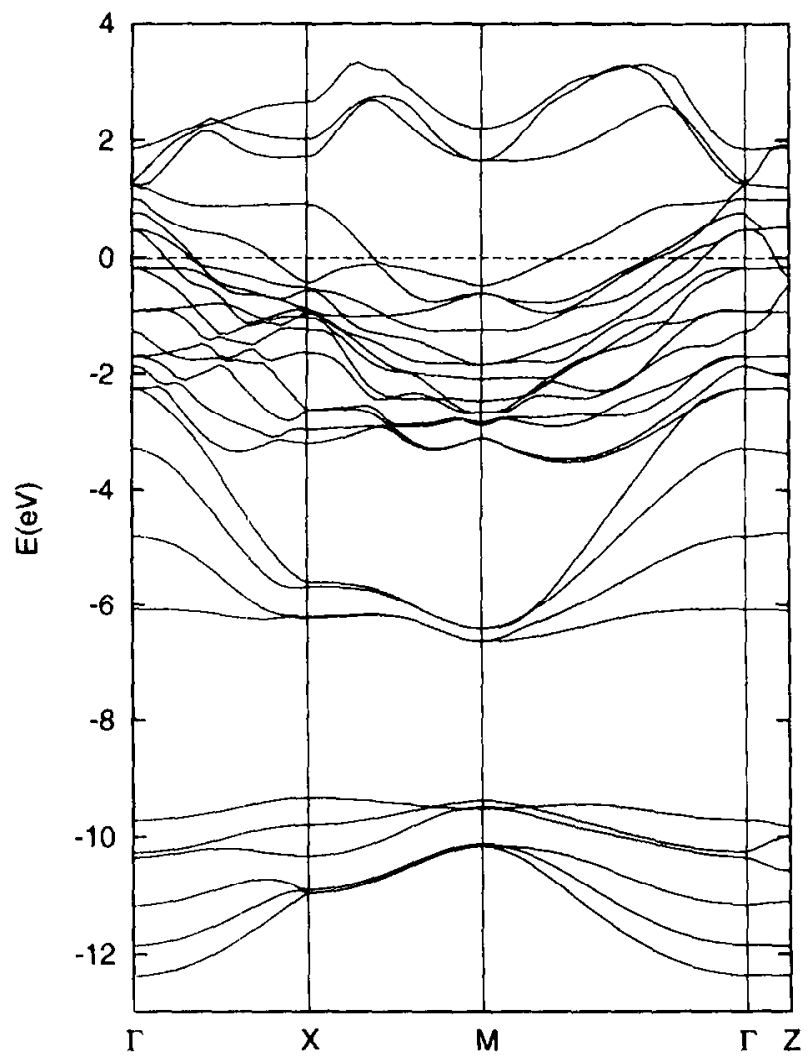

Fig. 3. Band structure of the (As) $)_{1}(\mathrm{GaAs})_{2} /(\mathrm{ErAs})_{1} /$ $(\text { AsGa })_{2}$ superlattice projected along symmetry directions of the two-dimensional BZ of the (100) surface and along the direction perpendicular to the surface $\mathrm{BZ}$, indicated as $\mathrm{Z}$. Energies (in $\mathrm{eV}$ ) are referred to the Fermi level of the system, shown by a dashed line. effect of the reduced dimensionality of the ErAs layer. However the interface states, which cross the Fermi level, are still present as a consequence of the unchanged interface geometry. It is worthwhile to mention that the presence and the energy localization of these partially filled interface bands depends strongly on the choice of the interface structure; the expected relaxation of the position of the interface As atoms is bound to change their energy. Besides a different bonding at the interface between REAs and GaAs will result in a different ordering of the states in the energy band structure; we will return to this point later.

As previously mentioned, Petukhov et al. [6] recently proved that, for bulk ErScAs, the inclusion of a quasiparticle self-energy correction could remove the crossing of the $\Delta_{2}^{\prime}$ and $\Delta_{5}$ bands due to the hybridization between As $4 p$ and Er $5 d$ electrons. Since Xia et al. [5] have argued that it is this crossing that inhibits the opening of a size quantization gap in thin layer of REAs embedded in GaAs we will now address this point.

In order to understand the different role of $\Delta$ crossing and of interface states regarding the lack of a semimetalsemiconductor transition in these superlattices, we have performed also a selfconsistent calculation of the electronic properties of a $(\mathrm{As})_{1}(\mathrm{GaAs})_{2} /(\mathrm{YbAs})_{1} /(\mathrm{AsGa})_{2}$ structure. Differently from $\mathrm{Er}$, the $\mathrm{RE}$ atom $\mathrm{Yb}$ has a fully occupied $4 f$ shell. Our choice of YbAs is motivated by the fact that in this case the compound bulk band structure shows no more $\Delta$ crossing and in any case the $\Delta$ lines are now well above the Fermi level [7]. This is true both for the case of treating in the calculations the $4 f$ electrons as core levels or including it as valence electrons $[7,8]$. Thus this arsenide compound is a good candidate for gap opening in a confined situation. We have chosen to treat the $f$ electrons as valence electrons because of their energy location; their inclusion in the YbAs bulk compound energy band structure gives rise to flat bands immediately below the Fermi level and, by hybridizing with the As $4 p$ electrons, two $\Gamma_{15}$ states repel each other. Our calculated energy band structure for the (As) $)_{1}(\mathrm{GaAs})_{2} /(\mathrm{YbAs})_{1} /(\mathrm{AsGa})_{2}$ superlattice is depicted in Fig. 4. It is evident that indeed a gap opens up in the band structure throughout the whole $\mathrm{BZ}$ between $\sim 1.5 \mathrm{eV}$ and $-1.7 \mathrm{eV}$; also the corresponding density of states shows a gap at these energies. Nevertheless the superlattice remains semimetallic, again as a consequence of the presence of the As interface atoms derived states. Therefore, although the structure is semimetallic, it is conceivable to induce a transition to a semiconductor by shifting the Fermi level. This could be obtained either by taking into account interface relaxation and strain effects or with a different lattice registry at the interface [11]: one can consider a mixed YbAs plane directly connected to the GaAs underlying plane or the YbAs layers can 


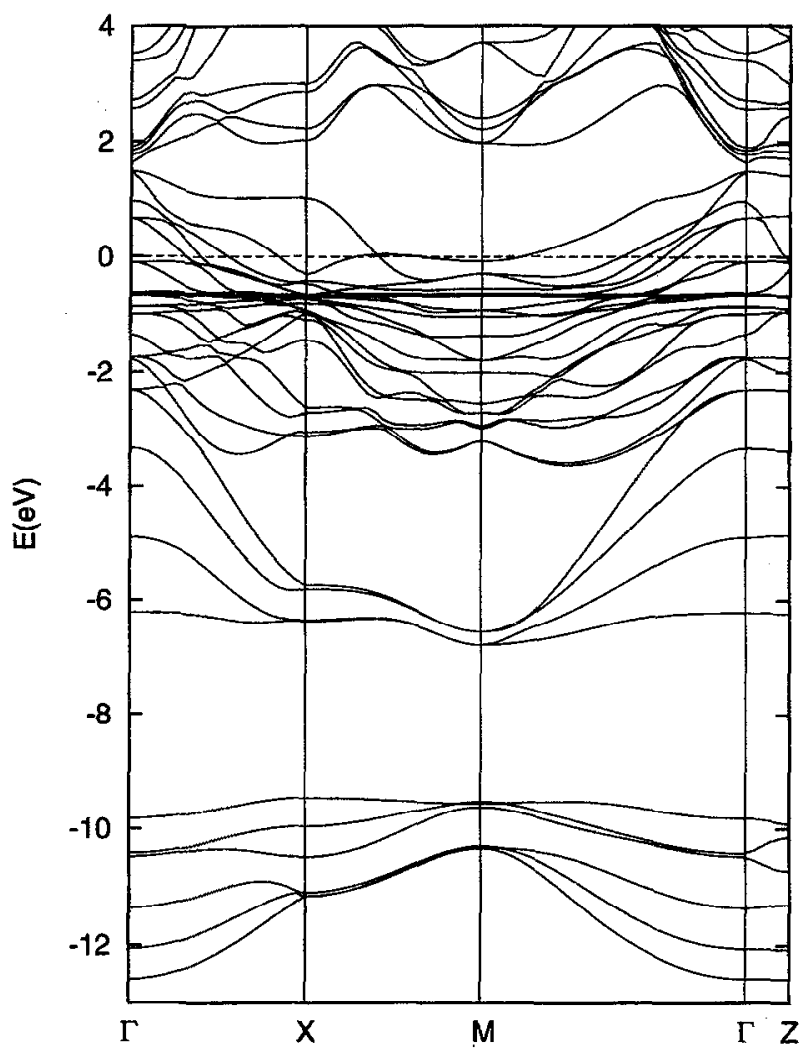

Fig. 4. Band structure of the (As) $)_{1}(\mathrm{GaAs})_{2} /(\mathrm{YbAs})_{1} /$ $(\text { AsGa })_{2}$ superlattice projected along symmetry directions of the two-dimensional $\mathrm{BZ}$ of the $(100)$ surface and along the direction perpendicular to the surface $\mathrm{BZ}$, indicated as $\mathrm{Z}$. Energies (in $\mathrm{eV}$ ) are referred to the Fermi level of the system, shown by a dashed line.

position themselves in the prolongation of the Ga planes; calculations for these different geometries are in progress. Moreover, there is the possibility to have some hydrogen atoms at the interface which saturate the unpaired $p$ electrons of the interface As atoms, removing the interface states and making the system semiconducting. A partial hydrogenation is also feasible in the experiments by depositing As under the form of arsine $\left(\mathrm{AsH}_{3}\right)$.

In conclusion, we have presented ab-initio band structure and density of states calculations of REAsGaAs superlattices. On the basis of our calculations, we conclude that the semimetal-semiconductor heterostructure is still semimetallic, the presence of interface states preventing the heterostructure from turning into a semiconductor. Different interface terminations or the presence of hydrogen atoms at the boundaries between the two systems can therefore have important consequences and we plan to study these effects in the future.

Acknowledgements-This work was also supported by Consiglio Nazionale delle Ricerche (CNR), Italy, and has benefitted from collaborations within the Network on " $A b$-initio (from electronic structure) calculation of complex processes in materials" (contract: ERBCHRXCT930369). For one of us (M.S.) this work was part of a HCM project of the EEC (Contract no. ERBCHBGCT930451). M.S. would like to thank N.E. Christensen and A. Svane for very useful discussions. The numerical computations was performed at the Centro Interdipartimentale di Calcolo Automatico e Informatica Applicata (CICAIA) Modena and at the Centro di Calcolo Elettronico dell' Italia Nord Orientale (CINECA) Bologna.

\section{REFERENCES}

1. Bogaerts, R., De Keyser, A., Herlach, F., Peeters, F.M., DeRosa, F., Palmstrom, C.J., Brehmer, D. and Allen S.J. Jr., Solid State Electron. 37, 1994, 789.

2. Allen, S.J. Jr., , Brehmer, D. and Palmstrom, C.J., in Rare Earth Doped Semiconductors (Edited by G.S. Pomrenke, P.B. Klein and D.W. Langer), Vol. 301, p. 307. Material Research Society, Pittsburgh, 1993.

3. Allen, S.J. Jr., DeRosa, F., Palmstrom, C.J. and Zrenner, A., Phys. Rev. B43, 1991, 9599.

4. Allen, S.J. Jr., Tabatabaie, N., Palmstrom, C.J., Mounier, S., Hull, G.W., Sands, T., DeRosa, F., Gilchrist, H.L. and Garrison, K.C., Surf. Sci. 228, $1990,13$.

5. Xia, J.-B., Ren, S.-F. and Chang, Y.-C., Phys. Rev. B43, 1991, 1692.

6. Pethukov, A.G., Lambrecht, W.R.L. and Segall, B., Phys. Rev. B50, 1994, 7800.

7. Said, M., Ossicini, S., Bertoni, C.M. and Fasolino, A. To be published.

8. Takegahara, K. and Kaneta, Y., J. Phys. Soc. Jpn. 60, 1991, 4009.

9. Palmstrom, C.J., Mounier, S., Finstad, T.G. and Miceli, P.F., Appl. Phys. Lett. 56, 1990, 382.

10. Guivarc'h, A., Guenais, B., Ballini, Y., Auvray, P., Caulet, J., Minier, M., Dupas, G., Ropars, G., Regreny, A., Guerin, R. and Deputier, S., J. Cryst. Growth 127, 1993, 638.

11. Lepine, B., Quemerais, A., Sebilleau, D., Jezequel, G., Agliz, D., Ballini, Y. and Guivarc'h, A. J. Appl. Phys. 76, 1994, 5218.

12. Stoffel, N.G., Palmstrom, C.J. and Wilkens, B.J., Nucl. Instrum. Methods B56, 1991, 792.

13. Guivarc'h, A., Ballini, Y., Minier, M., Guenais, B., Dupas, G., Ropars, G. and Regreny, A., J. Appl. Phys. 73, 1993, 8221.

14. A picture of the structure is given in [5] 